\title{
Wholes and Their Covers
}

\author{
Marcin Morzycki \\ University of Massachusetts Amherst
}

Adjectives such as whole and entire lend themselves quite readily to an intuitive characterization: What they have in common is, roughly, a requirement that a predicate hold of all the parts of an individual. This is straightforward enough, but underlying this initially clear intuition are puzzles that relate ultimately to part structure, distributivity, and quantification and scope mechanisms.

Despite this natural intuition, the argument advanced here is that DPs containing whole and entire - henceforth 'whole adjectives' - are obligatorily nonquantificational expressions. Their apparent quantificational force will be understood instead as a restriction on the tolerance normally extended to exceptions to a predication, building on Brisson's (1998) theory of nonmaximal interpretations of plurals. To implement this, the notion of covers (Schwarzschild 1996) will be imported, with some substantial modifications, from the semantics of plurals into the semantics of singulars, and a Brisson-style theory of nonmaximal interpretations of singulars will be developed on that basis. Along the way, the analysis will lead to adopting a particular conception of choice-function indefinites (Winter 1997, Reinhart 1997, Kratzer 1998, Matthewson 1999).

Section 1 makes some observations about whole adjectives, characterizing their interpretive effect in general terms, noting restrictions these adjectives impose on determiners and the anaphoric and scope properties they give rise to. Section 2 argues against analyzing whole adjectives as a species of universal quantifier, and then considers the highly intensional treatment proposed by Moltmann (1997). Section 3 advances an alternative analysis of whole adjectives as 'maximizing modifiers' in the spirit of Brisson, in the process extending the use of covers to the interpretation of singulars and finding reason to permit intermediate existential closure of choice functions (Winter 1997, Reinhart 1997). Section 4 concludes.

\section{Characteristics of Whole Adjectives}

\subsection{What Do They Do?}

The essential role of whole adjectives is to impose a requirement that no part of an individual be exempted from having to satisfy a predicate:

(1) a. The ferret is submerged.

b. The entire/whole ferret is submerged.

For (1a) to be true, it is under most circumstances quite sufficient for a significant proportion of the ferret to be submerged. If some stray fur or a tail protrudes above the surface of the water, one is not inclined to take this as evidence that (1a) is false. 
On the other hand, (1b) makes a stronger claim, one of total ferret submersion. Any unsubmerged ferret-matter, even if only a tail, renders (1b) false. So, to put this in slightly less atheoretical terms, one may conceive of the contribution of a whole adjective here as universal quantification over parts of the ferret or, alternatively, as a requirement that the tolerance that would otherwise be extended for a few exceptional unsubmerged ferret parts be withheld. These properties mirror those of all:

All of the ferret is submerged.

This seems to mean something very much like what (1b) means. More will be said about this parallel shortly.

Despite their superficial resemblance, whole adjectives differ from adjectives like complete or partial:

a. The entire/whole ferret is submerged.

b. The complete/partial ferret is submerged.

Unlike the whole adjectives in (3a), the complete adjectives in (3b) merely provide information about the structural integrity of the ferret. They are intersective, so ( $3 b)$ could be paraphrased as in (4):

(4) The ferret is submerged, and the ferret is complete/partial.

Thus (3b) and (4) are true if the ferret is submerged, more or less, and if it is a complete ferret, with all its ferret parts in the appropriate ferret configuration, or if it is a partial ferret, with not all of its ferret parts in the appropriate ferret configuration, respectively. Somewhat confusingly, whole actually has a reading as an adjective of this class, too; this is the only reading available for whole in predicative position, as in (5a). Entire, which is exclusively a whole adjective and lacks a complete adjective reading, cannot very naturally occur predicatively at all, as in (5b):

a. The ferret is whole.

b. ${ }^{*}$ The ferret is entire.

Whole adjectives, then, unlike complete adjectives, do not have property denotations.

There are nominal and adverbial relatives of whole adjectives, which, though of course relevant, will for the most part be set aside here:

a. The whole/entirety of the ferret is submerged.

b. The ferret is wholly/entirely submerged.

\subsection{Determiner Restrictions}

Whole adjectives may occur only with a restricted set of determiners. Definites, demonstratives, and genitives are fine: 

a. The whole/entire ferret is submerged.
b. This/that whole/entire ferret is submerged.
c. My/Floyd's whole/entire ferret is submerged.

So are DPs with indefinites headed by $a$, numerals, many, and less clearly some:
a. A whole/entire ferret is submerged.
b. Twenty whole/entire ferrets are submerged.
c. Many whole/entire ferrets are submerged.
d. Some whole/entire ferrets are submerged.

Strong, inherently quantificational determiners are systematically incompatible with whole adjectives. In these contexts, entire results in ungrammaticality, and whole receives only the complete adjective reading, which will be set aside here: ${ }^{1}$
a. *Every/each whole/entire ferret is submerged.
b. *All the whole/entire ferrets are submerged.
c. *Most whole/entire ferrets are submerged.

\subsection{Scope}

Whole adjectives appear to give rise to scope effects with respect to negation that would otherwise be absent:
a. The whole ferret wasn't submerged.
the wholef. $>\neg$; ᄀ> the whole f.
b. No one likes that whole subject. $\neg>$ that whole s.; that whole $s .>\neg$

In (10a), there seems to be a wide-scope reading of the whole ferret in which all of the ferret is claimed to have been unsubmerged, and a narrow-scope reading in which it is denied that all of the ferret was submerged. And (10b) can be used to deny that anyone likes all of that subject, or to assert that no one likes any of that subject. These effects are a bit striking, since definites and demonstratives do not normally participate in scope interactions. In this respect also, whole adjectives differ from complete adjectives, which do not give rise to scope ambiguities:
a. The complete ferret wasn't submerged.
b. Clyde didn't eat a partial sandwich.
unambiguous
unambiguous

This is less surprising, since complete adjectives have simple property denotations. indefinites:

Oddly, whole adjectives do not give rise to scope ambiguities with respect to

(12) a. The whole ferret was submerged by a child.

unambiguous

b. Someone ate this entire sandwich.

unambiguous

c. The entire committee complained about an especially

unambiguous 
misguided proposal.

One cannot judge (12a) true in a situation in which no single child is guilty of ferret submersion, but rather different children submerged different parts of the ferret. Likewise, (12b) cannot easily involve an act of cooperative sandwich consumption involving several people. Even a noun that provides a relatively clear and individuated part structure like committee in (12c) does not quite mitigate this effect (12c) requires a single, universally criticized proposal. With respect to existential quantifiers, then, whole adjectives do not seem to create additional scope possibilities.

This odd unwillingness to scope like normal, well-behaved universal quantifiers with respect to existentials is further reflected in the inability of whole adjectives to license the particular reading of different that arises only in the scope of universals (Beck 2000):

a. Each part of the ferret was submerged by a different child.

b. The whole ferret was submerged by a different child.

In the scope of a universal different may receive a reading in which it requires that the individuals quantified over by the existential vary with those quantified over by the universal. Whole adjectives do not give rise to this reading - (13b), for example, does not have a reading in which children must vary with ferret parts.

\subsection{Discourse Anaphora}

Among the characteristic properties of universals is that they do not introduce discourse referents, and indeed make any introduced inside their scope inaccessible beyond it (Heim 1982, Kamp 1984, others). Whole adjectives do not have these properties. Unlike their quantificational determiner counterparts, whole adjectives do not impede the DP in which they occur from itself introducing a discourse referent:

a. Every ferret was submerged. * It didn't seem pleased.

b. A entire ferret was submerged. It didn't seem pleased.

a. Cockroaches ate each sandwich. *It had been sitting out too long.

b. Cockroaches ate a whole sandwich. It had been sitting out too long.

Although the universally-quantified every ferret and each sandwich do not introduce discourse referents, DPs with whole adjectives do.

Whole adjectives do not prevent discourse referents from being introduced inside their scope, either, again contrasting with well-behaved universals:

a. Every building with a faulty roof $_{i}$ was demolished. ${ }^{*} I t_{i}$ had been leaking. b. An entire building with a faulty roof $_{i}$ was demolished. $I t_{i}$ had been leaking.

a. Each recent movie was marred by an irritating soundtrack . $^{*}{ }^{*} t_{i}$ will probably be released on CD.

b. The whole movie was marred by an irritating soundtrack . $_{i} t_{i}$ will probably 
be released on $\mathrm{CD}$.

So, for example, every prevents a faulty roof from licensing discourse anaphora in (16a); in (16b), whole has no such effect.

\section{Universal Part Quantification and Moltmann's Intensional Alternative}

\subsection{A Background Point: Singulars, Parts, and Parts of Singulars}

Though certainly important to the issues at hand, the vexing problem of the nature of singular part structure will be sidestepped here. But a few words are in order. (See Morzycki 2002 for a more detailed discussion.)

Moltmann (1997) develops an articulated theory of this relation. She notes that though singular part structure lacks the formal properties that characterize plural and mass noun denotations, there are important empirical parallels. Plurals and mass nouns have relatively well-behaved part structures, reflecting a number of welldefined formal properties (cumulativity, distributivity/atomicity, transitivity, and extensionality). Singular count noun part structure is not so tidy, reflecting none of these properties. Despite the stark formal difference between singular count nouns and plural and mass nouns, though, there is evidence that a unified treatment of all three is required. Among the clearest reflections of this is in partitives, which can be constructed from singulars, plurals, and mass nouns, in all cases with parallel syntax and parallel semantics. To face this challenge, Moltmann relativizes every predicate in the grammar to a reference situation which determines part structure, and assumes that every argument position of every predicate is filled with a pair one of whose members is a reference situation. For current purposes, though, it will be sufficient to suppose that there is some part relation for count singulars $\leq_{s}(s$ for 'singular') which has the necessary properties. This is a simplifying assumption, essentially a placeholder for an articulated theory of singular part structure. Although it seems likely that some of the additional theoretical machinery appealed to momentarily namely, covers - may be able to replicate some of the context-sensitivity Moltmann achieves with reference situations, nothing here is intended to be a theory of the singular part-structure relation itself.

\subsection{Universal Part Quantification: The Idea}

The initial intuition about whole adjectives we began with was that they impose a requirement that a predicate hold of all the parts of an entity without exception. One can express this intuition quite simply and directly by analyzing whole adjectives as universal part quantifiers. Thus a sentence such as (1) could be interpreted as in (18):

$$
\begin{aligned}
& \text { [the whole/entire ferret is submerged } \rrbracket=\forall \mathrm{x}\left[\mathrm{x} \leq_{s} \mathrm{ly}[\mathrm{ferret}(\mathrm{y})] \rightarrow\right. \\
& \text { (not final) }
\end{aligned}
$$


For all its simplicity and naturalness, this approach runs afoul of many of the observations in section 1. On its own it does not explain the determiner restrictions whole adjectives impose, and it makes wrong predictions with respect to scope and discourse anaphora. If whole adjectives denote universal part quantifiers, they should interact scopally with existentials and license the relevant reading of different; but they do neither. Another consequence of this view is the prediction that whole adjectives should render discourse referents introduced in their scope inaccessible to discourse anaphora; again, they do not have this effect.

\subsection{A Conjunctive Revision to the Universal Part Quantification Approach}

As expressed in (18), then, the universal part quantification analysis does not seem to be getting much traction on the problem. A slight revision may help. One might take whole adjectives to contribute universal part quantification in a conjunctive way:

$$
\begin{aligned}
& \llbracket \text { The whole/entire ferret is submerged } \rrbracket=\operatorname{submerged}(\mathrm{iy}[\text { ferret }(\mathrm{y})]) \wedge \\
& \forall \mathrm{x}\left[\mathrm{x} \leq_{s} \mathrm{ty}[\text { ferret }(\mathrm{y})] \rightarrow \operatorname{submerged}(\mathrm{x})\right] \\
& \text { (not final) }
\end{aligned}
$$

This reflects a paraphrase such as 'the whole ferret was submerged, and every part of the ferret was submerged (as well)'.

This revision immediately avoids the discourse anaphora trouble encountered above because one of the conjuncts is interpreted as though whole were absent and therefore unable to block discourse anaphora. This may initially appear to avoid the scope difficulties too, since any existentials that might occur in the first conjunct would not have any universal introduced by whole to interact with. But scope problems with indefinites would apparently remain. In order to confront this problem, it is necessary to consider more seriously how denotations such as (19) are to be assembled. Another example will be helpful:

(20) A painter moistened the whole canvas.

a. 【the whole canvas $\rrbracket=\lambda \mathrm{P} . \mathrm{P}(\mathrm{vy}[\operatorname{canvas}(\mathrm{y})]) \wedge \forall \mathrm{z}\left[\mathrm{z} \leq_{s} \mathrm{ly}[\operatorname{canvas}(\mathrm{y})] \rightarrow \mathrm{P}(\mathrm{z})\right]$

b. 【a painter 1 [the whole canvas ${ }_{2}\left[t_{1}\right.$ moistened $\left.\left.t_{2}\right]\right] \rrbracket=\exists \mathrm{x}[\operatorname{painter}(\mathrm{x}) \wedge$ $\operatorname{moisten}(\mathrm{ly}[\operatorname{canvas}(\mathrm{y})])(\mathrm{x}) \wedge \forall \mathrm{z}\left[\mathrm{z} \leq_{s} \mathrm{ly}[\right.$ ferret(y)] $\left.\rightarrow \operatorname{moisten}(\mathrm{z})(\mathrm{x})]\right]$

c. 【the whole canvas 2 [a painter moistened $\left.t_{2}\right] \rrbracket=\exists x[$ painter $(\mathrm{x}) \wedge$ $\operatorname{moisten}(\mathrm{ly}[\operatorname{canvas}(\mathrm{y})])(\mathrm{x})] \wedge \forall \mathrm{z}\left[\mathrm{z} \leq_{s} \mathrm{ly}[\right.$ ferret(y)] $\rightarrow \exists \mathrm{x}[\operatorname{painter}(\mathrm{x}) \wedge$ $\operatorname{moisten}(\mathrm{z})(\mathrm{x})]]$

The denotation of the whole canvas in (20a) encodes the conjunctive meaning. Crucially, this is a generalized quantifier denotation. Thus to be interpreted, the whole canvas will have to $\mathrm{QR}$ from object position, giving rise to LFs in which it takes scope somewhere below a painter, as in (20b), or above it, as in (20c). The denotation in (20b) does not present any obvious problems - it requires that there be some painter who moistened the canvas and who moistened every part of it. But the denotation in (20c) is too weak - it requires only that there be a painter who moistened the canvas, and that each part of the canvas had been painter-moistened. 
On this reading, then, this sentence would be true if a team of painters collectively moistened every part of the canvas, but no single painter moistened every part of it. This is not a possible reading.

It is not clear how this result could be avoided. So long as a whole adjective is attributed any quantificational force of its own with scope over the matrix predicate, even in this conjunctive way, the DP in which it occurs will have to denote a generalized quantifier and consequently be susceptible to $\mathrm{QR}$, and to the possibility of taking wide scope over an indefinite. The option of simply stipulating that DPs with whole adjectives do not $\mathrm{QR}$ is unavailable, since generalized quantifiers often must $\mathrm{QR}$ to be interpreted. One could stipulate that a whole $\mathrm{DP}$ can $\mathrm{QR}$ only to positions in which its relative scope is unaltered, but this is quite unappealing.

\subsection{Moltmann's Intensional Approach}

Moltmann (1997) proposes an analysis of whole along different lines, cast in her general theory of part structure. She proposes that the grammar is intricately sensitive to reference situations, or situations that determine the part structure an individual is construed as having. Whole, on this view, restricts an individual's perceived part structure, triggering an interpretation in which an individual is not construed as what she calls an 'integrated whole'. It applies to a pair of a reference situation and a situated generalized quantifier - a generalized quantifier interpreted with respect to a reference situation - and yields a situated generalized quantifier:

(21) Let Q be a situated generalized quantifier and s' a (Moltmann 1997, p. 81) situation:

$\llbracket$ whole $\rrbracket\left(\mathrm{Q}, \mathrm{s}^{\prime}\right)=\lambda \mathrm{s} \lambda \mathrm{P}\left[\mathrm{Q}^{\mathrm{s}^{\prime \prime}}\left(\mathrm{P}^{\mathrm{s}}\right)=1 \wedge(\forall \mathrm{x})\left[\mathrm{P}^{\mathrm{s}}\left(\mathrm{x}, \mathrm{s}^{\prime}\right)=1 \rightarrow\right.\right.$ BASED-ON $\left(<\mathrm{P}^{\mathrm{s}}\right.$, $\left.\left.\left.<\mathrm{x}, \mathrm{s}^{\prime}>>,<(\neg \mathrm{INT}-\mathrm{WH})^{\mathrm{s} "},<\mathrm{x}, \mathrm{s}^{\prime \prime}>>\right) \wedge \forall \mathrm{x}^{\prime}\left[\mathrm{x}^{\prime}<\mathrm{x} \rightarrow \mathrm{x}^{\prime}<\mathrm{s}\right]\right]\right]$, where s' is the situation that differs minimally from $s^{\prime}$ in that for all $x \in D\left(s^{\prime \prime}\right)$, $\neg$ INT-WH(x,s')

Superscripts indicate reference situations with respect to which predicates are evaluated; INT-WH(X,S) $=1$ iff $\mathrm{x}$ is an integrated whole in $\mathrm{S}$; BASED$\mathrm{ON}\left(<\mathrm{P}^{\mathrm{s}},<\mathrm{x}, \mathrm{s}^{\prime}>>,<\mathrm{Q}^{\mathrm{s}},<\mathrm{y}, \mathrm{s}^{\prime \prime},>>\right)=1$ iff $\mathrm{P}^{\mathrm{s}}\left(<\mathrm{x}, \mathrm{s}^{\prime}>\right)$ 'forms a basis of' $\mathrm{Q}^{\mathrm{s} \text { " }}\left(<\mathrm{y}, \mathrm{s}^{\prime \prime},>\right)$. Paraphrasing what (21) is intended to express is not entirely straightforward. But its effect is in part that whole applied to the ferret with respect to a reference situation s' will yield a situated generalized quantifier true of is submerged with respect to a reference situation $s$ if the non-integrated-whole version of the ferret in the reference situation s' is submerged in s. The most important aspect of this for current purposes is that it gives rise to an interpretation in which an individual is construed as an amalgamation of parts, having a part structure that licenses distributivity.

Although Moltmann does not make this explicit, this appears to make the right predictions with respect to the scope and discourse anaphora facts. The crucial feature of (21) that makes this possible is the absence of any universal part quantifier with free variables in its scope. Because of this, no discourse referents could be introduced inside the scope of a universal part quantifier contributed by whole, where 
they would wrongly be predicted to be inaccessible. Similarly, no scope ambiguities are expected with respect to other quantifiers, because they could not occur inside the scope of any universal part quantifier. No scope ambiguities are predicted with respect to negation, either, but another explanation of these facts is in principle available, as section 4.11 will suggest. One immediately striking feature of the denotation in (21), though, is that it is not compositional. Whole applies directly to a DP denotation, though the overt position of whole adjectives in English is lower, below the $\mathrm{D}$ level. Moreover, without further elaboration, the ban on inherently quantificational determiners whole adjectives impose remains mysterious. Neither of these problems seems intractable. However, the intensional machinery necessary to execute Moltmann's general program is powerful, and its consequences for the grammar pervasive. In light of that, it might be desirable to consider alternatives in which the lexical semantics of whole need not be crucially intensional, and the necessary theoretical commitments need not be so many and so profound.

\section{Whole Adjectives as Maximizing Modifiers}

\subsection{Nonmaximal Interpretations: Brisson (1998)}

An alternative intuition about whole adjectives is available - namely, that they restrict exception tolerance. Brisson (1998) develops an analysis of plural all rooted in a corresponding intuition. This will provide the starting point here. For Brisson, all is a 'maximizing modifier' rather than a universal quantifier, in that it eliminates the ability of plurals to tolerate nonmaximal interpretations. Under normal circumstances, this tolerance is readily extended. One would normally judge The boys are building a raft true if only 98 of 100 boys are building a raft - this is a 'nonmaximal' interpretation. But not so for e.g. All the boys are building a raft where the presence of all eliminates this possibility. With all, no exceptions are tolerated.

\subsection{Covers}

To capture this effect, Brisson develops a model of nonmaximality in terms of a notion of a 'cover' of the domain of discourse (Schwarzschild 1996). A cover is essentially a means of structuring the domain so that various individuals are grouped together in a way consistent with the context. This is useful in accounting for interpretations of predicates that involve distributivity only to certain pragmaticallydetermined non-atomic parts. On such a reading, (22) - due to Gillon (1987) - is true of the Broadway musical writers Rodgers, Hammerstein, and Hart:

The men wrote musicals.

Rodgers and Hammerstein wrote musicals together, as have Rodgers and Hart. None of them, however, has written a musical alone, so the reading in question is not one that distributes to singular individuals. ${ }^{2}$ Nor is it the case that all three of the them 
wrote a musical together, so the reading in question is not a collective one, either. Covers provide a means of representing such readings. On the true reading, (22) is interpreted with respect to a cover which groups together Rodgers and Hammerstein, as well as Rodgers and Hart, but no other combinations of the three.

Put more precisely, a cover groups the individuals in a domain into cells. Thus, in the cover required to render (22) true, there is a cell containing only Rodgers and Hammerstein, and one containing only Rodgers and Hart, but none containing, for example, only Rodgers or only Hammerstein and Hart. The true reading now can be understood as a distributive one, but not in the conventional sense that involves distributivity to singular individuals; rather, distributivity here is to cells of the cover.

Schwarzschild expresses this in terms of a theory in which it is of central importance that plurals simply denote sets, rather than plural individuals in the sense of Link (1983). The notion of a cover, though, does not seem inextricably bound up in this decision, and it will be useful here to attempt separating them. Putting things in more Linkian terms, or at least in terms more compatible with a Link-style approach, one might suppose that each 'cell' of the cover is an individual, frequently a plural individual. A cover, then, is a set of individuals, singular or plural, which has the same supremum as the domain:

\section{Cov is a cover of D iff $\sup (\mathrm{Cov})=\sup (\mathrm{D})$}

That is, that the smallest plural individual composed of all the cells of the Cov must also be the smallest plural individual composed of all the members of $D$. Consequently Cov merely imposes a structure on D, adding nothing and leaving nothing out. Putting this to use, a cover such as the one in (24) will be required to render (22) true given the domain D indicated:

$\{$ Rodgers, Hammerstein, Hart, Greta, Hilda $\subseteq$ D, and D contains nothing else apart from all parts and all sums of its members Cov $=\{$ Rodgers + Hammerstein, Rodgers + Hart, Greta, Hilda $\}$

The nature of the part relation that undergirds $(23-24)$ will be left unexplored, though for the moment it will do no ham to suppose that it is Link's individual part relation.

To derive the desired reading for (22), these cells in the cover will have to be distributed over. Thus Schwarzschild proposes a VP distributivity operator Part, which is restricted by a cover variable Cov. Reformulating this in terms consistent with (23):

$$
\llbracket \operatorname{Part}_{C o v} \rrbracket^{\mathrm{g}}=\lambda \mathrm{P} \lambda \mathrm{x} \forall \mathrm{y}[[\mathrm{y} \in \mathrm{g}(C o v) \wedge \mathrm{y} \leq \mathrm{x}] \rightarrow \mathrm{P}(\mathrm{y})]
$$

(not final)

What this requires is that a predicate to which this distributivity operator applies be satisfied by every cell in the cover which is a part of the individual distributed over. The relevant reading of (22), then, could be derived as in (26):

The men wrote musicals.

$\llbracket$ Part $_{C o v} \rrbracket(\llbracket$ wrote musicals $\rrbracket)(\llbracket$ the men $\rrbracket)=$ 
[Part Cov $\rrbracket($ wrote-musicals)(Rodgers + Hammerstein+Hart) $=$

$\forall \mathrm{y}[[\mathrm{y} \in \operatorname{Cov} \wedge \mathrm{y} \leq$ Rodgers + Hammerstein + Hart $] \rightarrow$ wrote-musicals $(\mathrm{y})]$

Given the cover in (24), it will in fact be the case that every cell that is a part of $\llbracket$ the

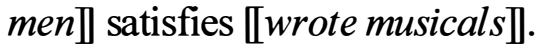

\subsection{Nonmaximality and Ill-Fitting Covers}

In this framework, Brisson points out, nonmaximal readings are expected. In the cover in (24), there are no coed cells - the men and the women are separated. But this is not necessary. One might imagine another kind of cover:

(27) \{Rodgers, Hammerstein, Hart, Greta, Hilda $\} \subseteq$ D, and D contains nothing else apart from all parts and all sums of its members

$\operatorname{Cov}=\{$ Rodgers + Hammerstein, Rodgers, Hart + Greta, Hilda $\}$

With respect to this cover, Hart need not have participated in any way in writing a musical for The men wrote musicals to be true. This is because Hart is not a member of the cover and so does not satisfy the restriction on the distributive operator. Of course, the plural individual Hart+Greta does, since this individual is a member of the cover - however, Hart+Greta is not a part of $\llbracket t h e$ men $\rrbracket$. Thus Hart may be an exception to the predication here, and a nonmaximal interpretation is permitted. Naturally, with only three men such an interpretation is pragmatically rather unlikely, for reasons Brisson discusses. ${ }^{3}$ But it is possible, and even more clearly when more men are involved.

Nonmaximal interpretations, then, arise with covers that are, in Brisson's terminology, ill-fitting. A cover is ill-fitting with respect to an individual $\mathrm{x}$ if there are parts of $\mathrm{x}$ that are stranded in cells in the cover that are not themselves parts of $\mathrm{x}$. More precisely, Brisson (1998: p. 97) proposes the definition like that in (28), reformulated here to adjust for different assumptions about plural denotations:

(28) For some cover of the universe of discourse Cov and some DP denotation $x$, Cov is a good fit with respect to $\mathrm{x}$ iff $\forall \mathrm{y}[\mathrm{y} \leq \mathrm{x} \rightarrow \exists \mathrm{z}[\mathrm{z} \in \operatorname{Cov} \wedge \mathrm{y} \leq \mathrm{z} \wedge \mathrm{z} \leq \mathrm{x}]]$

An ill-fitting cover is one that is not a good fit.

\subsection{Brisson's All and the Good-Fit Requirement}

Brisson proposes that what all does is impose a good-fit requirement on the cover with respect to which it is interpreted. This, of course, has the effect of eliminating the possibility of nonmaximal interpretations.

For Brisson, the way all makes this contribution is to restrict the range of possible assignment functions to only those with good-fitting covers. This, she suggests, is not part of truth-conditional meaning, but rather a component of what she 
calls the 'domain-adjusting meaning' of a sentence, an independent kind of meaning that is 'derived alongside truth conditions' (Brisson 1998: p. 210). An alternative approach to this, though, is to impose the good-fit requirement directly in the denotations of particular lexical items. So, in order to express the intuition that all maps a DP denotation onto itself and adds the requirement that it be interpreted with respect to a good-fitting cover, one might adopt a denotation such as (29):

$$
\left.\llbracket \text { all }_{C o v} \rrbracket^{\mathrm{g}}=\lambda \mathrm{x} \text { vy[y=x and } \mathrm{g}(\mathrm{Cov}) \text { is a good fit with respect to } \mathrm{x}\right]
$$

This amounts to treating the good-fit requirement as a presupposition (a possibility Brisson considers). To give the denotation of all access to the Cov variable with respect to which the DP will be interpreted, it is subscripted with it here. This sort of access is apparently required in the domain-adjusting meaning approach too. It is not obvious how to ensure that the cover variable with which all is subscripted matches the cover variable with which the corresponding Part operator is subscripted. This will have to remain a stipulation, though one might certainly imagine that some binding-theoretic requirement is involved. Importantly, this denotation - as well as any built on Brisson's definition of a good fit - will require that the DP argument of all be of type e, since the part relation is defined for this type only.

\subsection{The Singular Good Fit Requirement and Whole Adjectives}

To extend this approach to whole adjectives, a number of additional revisions will be necessary. The most basic of these will be to make reference to parts of singulars in the definition a good fit:

(30) For some cover of the universe of discourse Cov and some DP denotation $\mathrm{x}$, Cov is a singular good fit with respect to $\mathrm{x}$ iff $\forall \mathrm{y}\left[\mathrm{y} \leq_{s} \mathrm{x} \rightarrow \exists \mathrm{z}\left[\mathrm{z} \in \operatorname{Cov} \wedge \mathrm{y} \leq_{s} \mathrm{z}\right.\right.$ $\left.\left.\wedge \mathrm{z} \leq_{s} \mathrm{x}\right]\right]$

A singular counterpart would have to be defined for the Part operator as well, again by simply replacing the plural part relation with the singular one. A tentative definition of whole (and the apparently synonymous entire) can now be provided:

$$
\begin{aligned}
& \llbracket w_{\text {hole }} \text { Cov } \rrbracket=\lambda \mathrm{x} \cdot \mathrm{g}(\mathrm{Cov}) \text { is a singular good fit with respect } \\
& \text { to } \mathrm{x} \\
& \text { (not final) }
\end{aligned}
$$

\subsection{The Determiner Restriction}

Given (31) as it stands, there is no reason to expect the incompatibility of whole adjectives with inherently quantificational determiners. This will be corrected here by assuming a relatively high type - a brute-force technique, but in this instance perhaps an explanatory one. Rather than denoting properties as in (31), whole adjectives can take determiner denotations as arguments: 


$$
\llbracket \text { whole }_{C o v} \rrbracket^{\mathrm{g}}=\lambda \mathrm{P} \lambda \mathrm{D} . \mathrm{x}[\mathrm{x}=\mathrm{D}(\mathrm{P}) \wedge \mathrm{g}(\mathrm{Cov}) \text { is a singular good fit w.r.t. } \mathrm{x}]
$$

This yields the unique individual that the DP would have denoted had the whole adjective been absent, with the additional requirement that the DP be interpreted with respect to a good-fitting singular cover. Thus it must be the case that had the whole adjective been absent, the DP denotation would have been of type e. Inherently quantificational determiners, then, are ruled out.

This approach has at least three virtues, aside from empirical adequacy. First, it parallels very closely the denotation given for all in (29). This is more than a superficial or accidental parallel - it amounts to the claim that whole adjectives are incompatible with inherently quantificational determiners for the same reason that all is incompatible with inherently quantificational DP complements. Yet the high type allows this to be captured without being forced into syntactic assumptions, such as movement of whole adjectives to a position above the DP. Second, because in (32) whole adjectives no longer denote properties, the correct prediction is made that they should not occur in predicative positions. Third, in supposing that whole adjectives take determiner denotations as arguments, something is reflected of the special relationship certain 'functional' kinds of adjectives may bear to determiners. Larson (1999) and Zimmermann (2000) have approached this by proposing that certain adjectives may incorporate with certain determiners. It seems desirable, then, to be able to capture a close relationship of this sort. Yet, aside from the similarity to all, it is not obvious that whole adjectives in English incorporate in a syntactic or morphological sense. Supposing that whole adjectives take determiner arguments provides a way of expressing this relationship without requiring these other commitments. In this case, then, the higher-type approach may actually be more desirable than a lower-type alternative might be. ${ }^{4}$

\subsection{Choice Functions}

There is a certain complication the denotation in (32) presents. It requires that DPs modified by whole adjectives denote individuals. This is straightforward for definites. But for indefinites, especially nonspecific ones, further assumptions must be made. Given the current denotation of whole, any DP modified by a whole adjective must be of individual-denoting. For specific and wide-scope indefinites, this can be achieved naturally through a choice function interpretation (Winter 1997, Reinhart 1997, Kratzer 1998, Matthewson 1999). ${ }^{5}$ Thus, assuming the choice-function interpretation for $a$ sandwich in (33a), a whole sandwich may have the denotation in (33b):

a. $\llbracket a \rrbracket(\llbracket$ sandwich $\rrbracket)=\mathrm{f}($ sandwich $)$, where $\mathrm{f}$ is a partial function picking out an $\mathrm{x}$ such that $\mathrm{P}(\mathrm{x})$

b. $\llbracket$ whole $_{C o v} \rrbracket^{\mathrm{g}}(\llbracket$ sandwich $\rrbracket)(\llbracket a \rrbracket)=\mathrm{ix}[\mathrm{x}=\mathrm{f}($ sandwich $) \wedge \mathrm{g}(\mathrm{Cov})$ is a singular good fit w.r.t. x]

In light of the denotation in (32), every indefinite modified by whole must be interpreted this way. This requires particular assumptions about choice functions. 
In particular, the choice function mechanism must be one that could permit indefinites modified by whole adjectives to take narrow scope, as in (34):

Every ferret ate an entire sandwich.

This has a reading in which sandwiches eaten vary with ferrets. In order to accommodate this, it will be necessary to assume that choice functions may be existentially closed inside the scope of other quantifiers (Winter 1997, Reinhart 1997). Supposing choice functions receive only widest-scope existential closure (Matthewson 1999) would predict that the indefinite in (34) should have only a widescope reading. Although it is not entirely straightforward to demonstrate, a parameterized choice function mechanism of the sort proposed by Kratzer (1998) may not suffice, either. Kratzer accounts for pseudoscope effects - cases where indefinites take unexpectedly wide scope (or, in some cases, unexpectedly narrow scope) - by proposing that indefinite determiners may denote choice functions with an implicit argument that can be bound by higher quantifiers, creating the illusion of unexpected scope. This could in principle account for (34). It might be the case that the indefinite determiner here denotes a choice function with an argument bound by every ferret, so that for each ferret there is a different way of picking out a particular sandwich. But Kratzer further proposes that choice functions have values provided by the context - essentially, that only specific indefinites may have choice-function interpretations. Yet one may probably utter (34) on the reading in which sandwiches may vary with ferrets in a context that does not make salient a particular function from ferrets to sandwiches.

\subsection{Trouble with Indefinites}

At this point, there are some bumps in the road. As it stands, the account predicts certain interpretations of indefinites in embedded clauses that are not in fact possible. ${ }^{6}$

The crucial fact about the interaction of whole-adjectives and indefinites is that there doesn't seem to be any. Yet the Part distributivity operator introduces a universal quantifier with a property variable free in its scope, leaving a backdoor open through which existential quantifiers might sneak in and perniciously achieve narrow scope. In monoclausal sentences, one might guard against this risk by supposing that $\mathrm{QR}$ can attach indefinites only to proposition- or truth-value denoting expressions; a corresponding assumption might be maintained on the choice-function approach adopted above. The Part operator, on the other hand, applies only to predicates of individuals, so in monoclausal structures it will necessarily occur below the lowest point at which an indefinite may be interpreted, thereby ensuring that no indefinite is interpreted inside its scope.

But in complex sentences, this mode of explanation is unavailable, and the backdoor is wide open. Before facing the problem head-on, though, it may be helpful to demonstrate that indefinites introduced in embedded clauses indeed do not seem to be in the scope of a universal even when a whole DP occurs in the matrix clause. Contrasts in the available interpretations of different reflect this especially clearly: 
a. Every committee member suggested that Floyd submerge a different ferret.

b. The whole committee suggested that Floyd submerge a different ferret.

a. Everyone in town suspects that a different lamp store is run by the mafia.

b. The whole town suspects that a different lamp store is run by the mafia.

Just as when whole and different occur in clausemate DPs, the particular reading of different that arises in the scope of universals is not present in the (b) sentences, as it is in the (a) sentences. The embedded indefinites, then, are not in the scope of a universal. $^{7}$ Yet as the account now stands, they should be. If the embedded indefinite scopes within its clause, it would be inside the scope of the universally-quantifying Part operator. Illustrating:

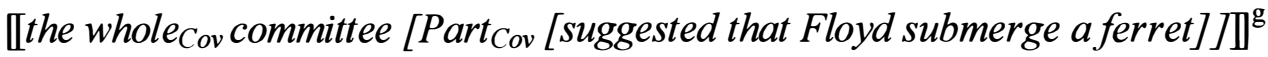

$=\forall \mathrm{y}\left[\left[\mathrm{y} \in \mathrm{g}(C o v) \wedge \mathrm{y} \leq_{s} \mathrm{~lx}[\mathrm{x}=\mathrm{tz}[\right.\right.$ committee $(\mathrm{z})] \wedge \mathrm{g}($ Cov $)$ is a singular good fit w.r.t. $\mathrm{x}]] \rightarrow \operatorname{suggest}\left(\wedge^{\wedge} \exists[\mathrm{f}\right.$ submerge $(\mathrm{f}(\mathrm{ferret}))($ Floyd $\left.\left.)]\right)(\mathrm{y})\right]$

Since the existential scopes under the universal, this reading of different is predicted.

\subsection{The Singular Part Operator}

This scope problem relates to a more general qualm one might have about using a Part distributivity operator in the interpretation of singulars: it places some notion of distributivity, however weakened by the introduction of covers, foursquare at the heart of the semantics of singulars. This on its own seems suspicious. Perhaps, then, the Part operator is to blame for the troubles. We cannot simply throw out the rotten apple here, however. While distributivity may not be quite what is required, the Part operator does more than that. It is also crucial to the mechanics of covers - it does the essential work of relating a predicate and an individual in a cover-sensitive way. What is necessary, then, is some means of doing this essential work without distributivity. Fortunately, this seems to be possible.

Rather than simply borrowing the singular Part operator as a kind of calque on the plural one, one can define the singular Part operator on its own terms. First, though, it may help to reflect more generally about what it means to predicate something of a singular. If I assert of a sandwich that it is eaten, I seem to be predicating consumption in a kind of collective way of a single individual, possibly an 'integrated whole' in Moltmann's sense. Depending on the context, this individual may not actually include every part of the sandwich, of course. But I am probably not predicating consumption in some independent, distributive way of the bread, the hummus, the cucumber slices, and the mysterious little seeds (cumin?) falling all over my plate. Indeed, if I had wanted to do this, I might have used a plural. We can perhaps do justice to this vague intuition by supposing that the singular Part operator, unlike its plural counterpart, applies a predicate to only one cell in the cover namely, in this case, the one containing whatever I regard as constituting the sandwich. Assuming this context is fairly normal, the bread, hummus, cucumber 
slices, and most of the seeds will probably count as part of the sandwich, but the seeds that have fallen on my plate probably do not. Thus these sandwich-parts will make up a single cell of the cover; the fallen seeds will have to be included in some other cell of which eating will not be predicated. Things might look like this:

\{the bread, the hummus, the cucumbers slices, the seeds that have fallen, the seeds that have not fallen, me, my plate, Clyde $\} \subseteq \mathrm{D}$, and D contains nothing else apart from all parts and all sums of its members

$\mathrm{Cov}=\{$ the bread+the hummus + the cucumber slices + the seeds that have not fallen, the seeds that have fallen+my plate, me, Clyde\}

This reflects a nonmaximal reading - it separates the irrelevant parts of the sandwich - but does not involve distributivity. It is, in fact, precisely analogous to how collective predication works in plurals. In singulars, though, this seems to be the only interpretation possible. Use of a singular seems to presuppose that the cover contains a single cell of which something will be predicated.

The singular Part operator can be redefined to reflect this requirement; its plural counterpart is repeated here as well, and they are more clearly distinguished:

$$
\begin{aligned}
& \text { a. } \llbracket \operatorname{sgPart}_{C o v} \rrbracket^{\mathrm{g}}=\lambda \mathrm{P} \lambda \mathrm{x} . \mathrm{P}\left(\mathrm{ly}\left[\mathrm{y} \in \mathrm{g}(\text { Cov }) \wedge \mathrm{y} \leq_{s} \mathrm{x}\right]\right) \\
& \text { b. } \llbracket \text { plPart }_{C o v} \rrbracket^{\mathrm{g}}=\lambda \mathrm{P} \lambda \mathrm{x} . \forall \mathrm{y}[[\mathrm{y} \in \mathrm{g}(C o v) \wedge \mathrm{y} \leq \mathrm{x}] \rightarrow \mathrm{P}(\mathrm{y})]
\end{aligned}
$$

The singular Part operator is now no longer a distributivity operator. It applies its first argument, a property, to the unique cell in the cover that is part of its second argument, an individual. The plural Part operator is just as it was before.

The cover in (38) satisfies the presupposition of the singular Part operator, since there is a unique cell that contains only parts of the sandwich. A cover otherwise identical to it in which the seeds that have fallen are a cell of their own, however, would not, since there would be two sandwich-parts-only cells. That this possibility is ruled out in singular predication - understood here as a failure of presupposition - is consistent with the intuition expressed that singular predication is in a sense always collective. Despite this quite fundamental revision, the role of whole adjectives remains unchanged. They can still contribute a good fit requirement, just as they did before.

Now that the definitions of the singular and plural Part operators are so clearly distinct, it becomes especially pressing that the right Part operator occur with the right sort of DP. There are a number of ways of approaching this. One might imagine that mismatches are ruled out by what varieties of individuals can stand in the singular or plural relation to each other. Alternatively, this could be a matter of syntactic agreement. If, as Schwarzschild suggests, Part operators are related to floated quantifiers, this would not be unexpected - floated quantifiers may in fact agree with their associated DPs, as they do in for example Hebrew (Shlonsky 1991) and German (Merchant 1996). A related understanding might view the relationship between the plural Part operator and floated quantifiers as fundamentally the same as the relationship between the singular Part operator and adverbs that seem to quantify over parts of a singular, such as mostly, wholly, or largely, or even the use of all in 
expressions like all wet (cf. Schwarzschild 1996, p. 162-170). ${ }^{8}$

\subsection{Scope of Indefinites Revisited}

Eliminating universal quantification from the singular Part operator also eliminated the prediction of unattested scope interactions with respect to indefinites. The new interpretation of the previously problematic (37) illustrates this:

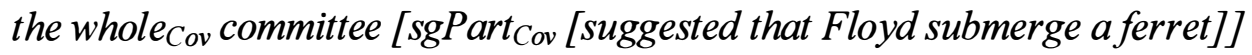

$$
\begin{aligned}
& \left.\llbracket \text { sgPart }_{\text {Cov }}[\text { suggested that Floyd submerge a ferret }]\right] \rrbracket^{\mathrm{g}}=\lambda \mathrm{x} \text {. } \\
& \text { suggest }(\wedge \exists \mathrm{f}[\operatorname{submerge}(\mathrm{f}(\text { ferret }))(\text { Floyd })])\left(\mathrm{vy}\left[\mathrm{y} \in \mathrm{g}(\text { Cov }) \wedge \mathrm{y} \leq_{s} \mathrm{x}\right]\right)
\end{aligned}
$$

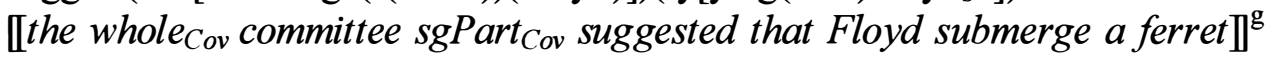

$$
\begin{aligned}
& =\operatorname{suggest}\left({ }^{\wedge} \exists \mathrm{f}[\operatorname{submerge}(\mathrm{f}(\text { ferret }))(\text { Floyd })]\right)(\mathrm{vy}[\mathrm{y} \in \mathrm{g}(C o v) \wedge \\
& \left.\left.\mathrm{y} \leq_{s} \mathrm{~lx}[\mathrm{x}=\mathrm{cz}[\operatorname{committee}(\mathrm{z})] \wedge \mathrm{g}(\mathrm{Cov}) \text { is a singular good fit w.r.t. } \mathrm{x}]\right]\right)
\end{aligned}
$$

The existential introduced by the indefinite was previously in the scope of the universal quantifier over cells in the cover. Now, there is no such universal, and no scope interactions with the Part operator are expected. More generally, an explanation has now emerged for why whole adjectives do not give rise to scope ambiguities with respect to indefinites. Whole adjectives are not themselves universally part-quantifying, and the singular Part operator does not contribute any universal quantifiers that might give rise to such scope interaction, either.

\subsection{Scope of Negation: Not, Scope, and Not Scope}

At this point, one might be concerned about the apparent scope interactions between whole adjectives and negation. In redefining the singular Part operator, we have eliminated scope interactions with indefinites, but in doing so, have we also lost an account of the interaction of whole adjectives and negation?

In one sense, yes. A scope-based account is no longer possible, since the Part operator no longer contributes a universal with respect to which negation could in principle scope. But there is another way of looking at things. In a sentence such as (41), the interpretation assigned is now a weak, scopally 'underspecified' one:

$$
\begin{aligned}
& \text { [the } \text { whole }_{\text {Cov }} \text { ferret isn't } \text { [sgPart }_{\text {Cov }} \quad{\text { submerged }] \rrbracket^{\mathrm{g}}}= \\
& \neg \text { submerged }\left(\mathrm { ly } \left[\mathrm{y} \in \mathrm{g}(\text { Cov }) \wedge \mathrm{y} \leq_{s} \mathrm{x}[\mathrm{x}=\mathrm{z}[\text { ferret }(\mathrm{z})] \wedge \mathrm{g}(\text { Cov }) \text { is a singular }\right.\right. \\
& \text { good fit w.r.t. } \mathrm{x}]])
\end{aligned}
$$

What is denied here is that the ferret, construed maximally, is submerged; it is not asserted that the ferret, construed maximally, is unsubmerged. For current purposes, this could actually be paraphrased in quantificational terms: this interpretation corresponds to a reading in which negation has scope over a universal part quantifier, not vice versa. Of course, such a weaker interpretation does not rule out the possibility that the stronger one could also be true. Perhaps, then, the weaker reading 
is all the semantics provides, and the stronger reading arises in some contexts pragmatically.

One reason this seems a sensible route to take is that some version of it would be necessary anyway in a scope-based account:

Floyd really didn't want to talk about that whole sensitive topic.

The most natural interpretation here is the strong one, in which this sentence is taken to claim that there was no part of the topic Floyd wanted to talk about. But even on a scope-based account, only the weaker interpretation (in which Floyd's desire was for discussion of not all of the topic) could be provided by the semantics. Both the whole adjective and the relevant singular Part operator are embedded under negation here, which would yield the weak reading. And because negation and the whole DP are not clausemates, even if some quantificational force could be assumed for the DP, it is not clear how it could achieve scope over the matrix-clause negation. It is independently necessary, then, to assume that stronger readings can sometimes arise for sentences in which the semantics provides only the weaker ones.

Another reason for attributing the stronger interpretations to pragmatic strengthening is their sensitivity to lexical properties of predicates. The stronger interpretations arise most naturally with what Yoon (1996; see also Kennedy and McNally 1999 and Rotstein and Winter 2001) calls partial predicates - very roughly, predicates such as dirty, wet, or touch that typically hold of an individual if they hold of any part of it. These contrast with total predicates such as clean, dry, or eat. ${ }^{9}$ Partial predicates give rise to the stronger reading more easily:

a. Clyde didn't touch/eat this whole sandwich.

b. Floyd didn't mention/solve that entire problem.

Out of the blue, it is quite natural to take the partial predicate versions of these as involving the complete absence of sandwich-touching and problem-mentioning. But the most likely interpretation of the total predicate sentences involves incomplete sandwich-consumption and incomplete problem-solving. The outlines of an explanation of this contrast may lie in the observation that on the weak reading, the partial-predicate sentences deny something independently unlikely. It is not normally informative to deny that Clyde made full physical contact with all of a sandwich or that Floyd's mere mention of a problem exhausted it entirely. It seems reasonable, then, that the stronger readings would generally be more salient. The weak readings of the total-predicate sentences, however, deny something relatively less pragmatically odd. Again, this is a case in which independent pragmatic factors probably must be appealed to on any approach.

\subsection{Discourse Anaphora}

The view that whole adjectives are maximizing modifiers rather than universal part quantifiers seems to provide a natural explanation of their discourse anaphoric 
properties. Since whole adjectives merely enforce a requirement on covers, with respect to discourse anaphora DPs containing them should behave though they were absent. This correctly predicts that whole adjectives should have no effect on discourse anaphora possibilities.

As noted in section 1, DPs with whole adjectives may introduce discourse referents, while universally quantified DPs may not. This is now expected, since there will be no universal quantifier in DPs modified by whole adjectives. For exactly the same reason, DPs with whole adjectives do not impede DPs in their scope from introducing discourse referents accessible outside it. Again, since whole adjectives are not universal quantifiers, and indeed are nonquantificational, it is expected that they should have no effect on discourse anaphora possibilities.

\subsection{Final Remarks}

At least one puzzle associated with whole adjectives that has not been addressed here - their relationship to polarity - bears briefly pointing out. One aspect of this is explored in den Dikken (to appear), who examines a negative polarity item incarnation of whole in Dutch (heel) with intricate and unusual licensing conditions. Interestingly, this heel occurs most easily with demonstratives, which may correspond to an effect in English in which demonstratives most easily seem to give rise to strengthened readings with respect to negation (Floyd doesn't want to talk about this/the/an entire subject). In German, a positive polarity item interpretation arises when the whole adjective occupies a pre-numeral position (Angelika Kratzer, p.c.). Although these issues will be left for further research, the use of covers seems a promising beginning in this respect. Covers are essentially a kind of domain restriction - and the good-fit requirement is an intellectual cousin to the domainwidening Kadmon and Landman (1993) suggest is responsible for the polarity sensitivity of English any - so these facts accord with what this sort of analytical path might lead us to independently.

To summarize, the central argument made here is that whole adjectives are not universally quantifying, but maximizing modifiers in the sense of Brisson (1998). This accounts for their essential semantic contribution, the restrictions they impose on determiners, their scope properties, and the anaphoric possibilities they license. In the course of pursuing this approach, a general model of nonmaximal interpretations of singulars was developed and some consequences for choice functions were encountered. To the extent that this approach managed to explain nonmaximal interpretations of singulars and plurals similarly using the same sorts of theoretical tools, it suggests that there is something right about the analytical intuition, expressed especially by Moltmann (1997), that part structure in singulars and in plurals should be understood in highly parallel terms. 


\section{Endnotes}

* Thanks to Angelika Kratzer, Lisa Matthewson, Barbara Partee, Ana Arregui, Meredith Landman, Anne-Michelle Tessier, Helen Majewski, the UMass semantics reading group, and the Spring 2000 UMass semantics seminar. Thanks also to the audience at SALT, and in particular to Roger Schwarzschild, Christine Brisson, Polly Jacobson, Gianluca Storto, and Bill Ladusaw. Naturally, remaining flaws are my responsibility. This paper is a highly abbreviated version of Morzycki (2002).

1 Though whole adjectives can occur with a definite determiner and they can occur with a plural noun, they cannot occur with both: *The entire ferrets were submerged. Also not very good is *?Some entire ferrets were submerged.

$2 \quad$ Since it would be misleading here, I will avoid the term 'atomic individuals'.

3 In fact, with the right sort of cover, any part of an individual, however small proportionally, can be sufficient for that individual to satisfy a predicate on a nonmaximal interpretation, modulo pragmatic restrictions (see Brisson 1998).

$4 \quad$ A similar determiner restriction is imposed by adjectives such as unspecified or unknown. Abusch and Rooth (1997) propose an account of this effect that hinges ultimately on the inability of quantificational DPs to introduce discourse referents that the adjective itself pick up. But it is not clear that this can extend to whole adjectives.

$5 \quad$ One might also attempt to draw the distinction between quantificational DPs and definites and indefinites in Kamp-Heim terms (Heim 1982, Kamp 1984).

6 Thanks especially to Roger Schwarzschild for making me think more clearly about scope with respect to indefinites.

7 Differences in available readings might be detectible here even without different, but different provides a convenient way of abstracting away from complications introduced by the independently present de re-de dicto ambiguity.

$8 \quad$ Thanks to a SALT reviewer for pointing out the relevance of these.

9 More might be said than is possible here about the relation between the partial/total predicate distinction and the cover approach to nonmaximal interpretations. One way of understanding partial predicates in this context is as predicates that generally tolerate extremely ill-fitting covers; total predicates can be understood to generally tolerate only covers that fit relatively well. Kennedy and McNally (1999) develop a fine-grained theory of essentially the partial/total distinction in adjectives in terms of their scale structure. Some connection between these ideas might be expected. Both approaches have at their heart the interaction of context-sensitivity with aspects of lexical semantics that can circumscribe it. Empirically, there is a connection, too. Certain part-structure adverbs that may be thought of as overt relatives of the singular Part operator (e.g. entirely, largely, mostly, wholly) also have readings that involve not proportions of an individual, but, loosely put, proportions of a scale. So The campus is completely nauseating is ambiguous - it may be that the campus has no non-nauseating parts, or else that it causes a maximal degree of nausea. Likewise, Oklahoma is wholly Republican and The table is largely dry manifest similar ambiguities. Further examination of this empirical connection, of the possible usefulness of covers in this domain, and of how this may relate to adjectival scale structure will have to be left to future research. 


\section{References}

Abusch, D. and M. Rooth. 1997. 'Epistemic NP Modifiers'. In A. Lawson, ed. SALT

7. CLC Publications, Ithaca, New York.

Brisson, C. 1998. Distributivity, Maximality, and Floating Quantifiers. Rutgers dissertation.

den Dikken, M. To appear. 'Direct and Parasitic Polarity Item Licensing'. The Journal of Comparative Germanic Linguistics.

Gillon, B. 1987. 'The Readings of Plural Noun Phrases in English'. Linguistics and Philosophy 13: 477-485.

Heim, I. 1982. The Semantics of Definite and Indefinite Noun Phrases. UMass dissertation.

Kadmon, N. and F. Landman. 1993. 'Any'. Linguistics and Philosophy 16.

Kennedy, C. and L. McNally. 1999. 'From Event Structure to Scale Structure: Degree Modification in Deverbal Adjectives.' In Mathews, T. and D. Strolovitch, eds. Proceedings of SALT LX. CLC Publications, Ithaca, NY.

Kratzer, A. 1998. 'Scope or Pseudo-Scope? Are There Wide-Scope Indefinites?'. In S. Rothstein, ed. Events in Grammar, 163-196. Kluwer, Dordrecht.

Larson, R. 1999. 'Semantics of Adjectival Modification'. Class notes, European Summer School in Logic, Language, and Information (ESSLLI).

Link, G. 1983. 'The Logical Analysis of Plurals and Mass Terms: A LatticeTheoretical Approach. In Bauerle, et al, eds. Meaning, Use, and Interpretation of Language. DeGruyter, Berlin.

Matthewson, L. 1999. 'On the Interpretation of Wide-Scope Indefinites'. Natural Language Semantics 7: 79-134. Kluwer, Dordrecht.

Merchant, J. 1996. 'Object scrambling and quantifier float in German'. In K. Kusumoto, ed., Proceedings of NELS 26, 179-193. UMass GLSA, Amherst.

Moltmann, F. 1997. Parts and Wholes in Semantics. Oxford, New York.

Morzycki, M. 2002. 'Maximality in the Interpretation of Singular DPs: Wholes Have Covers That Fit'. Ms, UMass Amherst.

Quine, W.V.O. 1960. Word and Object. MIT Press, Cambridge.

Reinhart, T. 1997. 'Quantifier Scope: How Labor is Divided Between QR and Choice Functions'. Linguistics and Philosophy 20, 335-397.

Rotstein, C. and Y. Winter. 2001. 'Partial Adjectives vs. Total Adjectives: Scale Structure and Higher-Order Modification'. Proceedings of the Amsterdam Colloquium.

Schwarzschild, R. 1996. Pluralities. Kluwer, Dordrecht.

Shlonsky, U. 1991. 'Quantifiers as functional heads: A study of quantifier float in Hebrew'. Lingua 84, 159-180.

Winter, Y. 1997. 'Choice Functions and the Scopal Semantics of Indefinites'. Linguistics and Philosophy 20,399-467.

Yoon, Y.. 1996. 'Total and Partial Predicates and the Weak and Strong Interpretations'. Natural Language Semantics 4: 217.236.

Zimmermann, M. 2000. 'Pluractional Quantifiers: The Occasional Construction in English and German'. In B. Jackson and T. Matthews, eds. Proceedings of SALT 10. CLC Publications, Ithaca, NY. 